\title{
MODELS AND REPRESENTATIONS FOR FAMILIES OF PARTS
}

\author{
Srinivas Raghothama \\ UGS, 10824 Hope St, \\ Cypress, CA 92804 USA \\ raghotha@ugs.com \\ Vadim Shapiro* \\ Spatial Automation Laboratory, \\ University of Wisconsin-Madison, \\ 1513 University Avenue, \\ Madison, WI 53706 USA \\ vshapiro@engr.wisc.edu
}

\begin{abstract}
A notion of class or family is fundamental in parametric modeling of mechanical parts. Due to lack of precise and formal characterization, parameteric families are usually defined in an ad-hoc fashion assuming specific representation schemes and implementations. This often results in ambiguous families, inconsistent transformatons between objects in the family, and difficulties in deciding whether a particular object belongs to the assumed family. These difficulties are further aggravated by lack of precise relationship and incompatibilities between any two such families.

We show that the notion of family can be formally defined as a category, and the relationship between any two families is properly stated in terms of a functor. Our formulation allows precise formulation of the problems in parametric modeling and gives insights into possible solutions and limitations. The proposed definition of a family is consistent with, but is more general than earlier proposals for families of sets in geometric tolerancing.
\end{abstract}

Keywords: Families of parts, Categories, Parametric modeling, Variational modeling 


\section{Motivation}

A notion of family is fundamental in parametric modeling of mechanical parts. Due to lack of precise and formal characterization, parameteric families are usually defined in an ad-hoc fashion assuming specific representation schemes and implementations. This often results in ambiguous families, inconsistent transformations between objects in the family, and difficulties in deciding whether a particular object belongs to the assumed family.

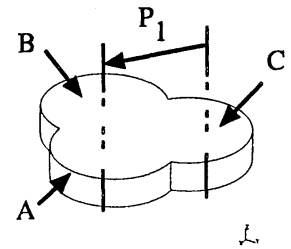

(a) $\mathrm{F}(\mathbf{P})=(\mathrm{A} \cup \mathrm{B}) \mathrm{U} \mathrm{C}$

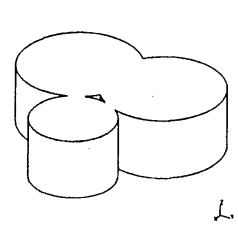

(b)

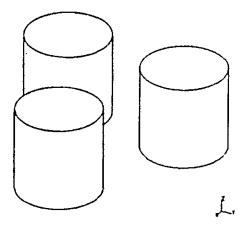

(c)

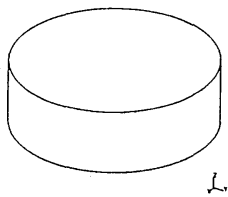

(d)

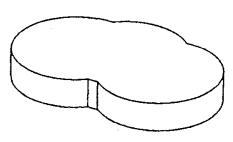

$L$

Figure 1. Family defined using a CSG representation

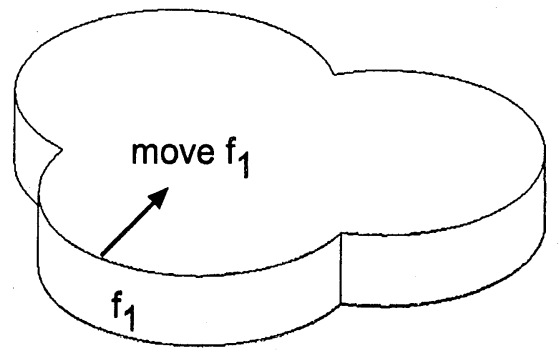

(a) B-rep K

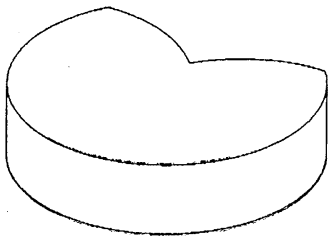

L

(b) B-rep L

Figure 2. Incorrect update for a b-rep "tweak" 
For example, a CSG family can be defined using a parametrized CSG representation. CSG families have a good high-level, parametric control, as illustrated by the example in figure 1. But they lack control over the shape (topological/geometrical properties) of the solid. The traditional approach for having shape control over the solid has been to define a family using b-reps and use local transforms or "tweaks" to modify them. For instance, figure 2 shows the b-rep $K$ corresponding to CSG $F(\mathbf{P})$ in figure 1(a). Transforming (tweaking) the cylindrical face $f_{1}$ by the transformation shown in figure 2(a), by a commercial solid modeling system results in the b-rep $L$ shown in figure 2(b), which is definitely not the expected result.

\section{Part families as categories}

The general idea of families of objects is captured by the well defined notion of categories in topology [Munkres, 1984; Jänich, 1983; Borisovich et al., 1985; Maunder, 1996]:

Definition 1 (Category of Objects) A category $\mathcal{C}$ consists of the following:

1 A class of objects $X$ called objects $(\mathrm{Ob})$ of the category.

2 For every ordered pair $(X, Y)$ of objects, a set hom $(X, Y)$ of morphisms $f$.

3 A law of composition hom $(X, Y) \times \operatorname{hom}(Y, Z) \rightarrow \operatorname{hom}(X, Z)$ for each triple of objects $(X, Y, Z)$.

4 If $f \in \operatorname{hom}(X, Y), g \in \operatorname{hom}(Y, Z)$ and $h \in \operatorname{hom}(Z, U)$ then, $h \circ(g \circ$ $f)=(h \circ g) \circ f .[$ Associativity]

5 For every object $X$ there is a morphism $1_{X} \in \operatorname{hom}(X, X)$ with the property $1_{X} \circ f=f$, and, $g \circ 1_{X}=g$, for all morphisms $f \in \operatorname{hom}(Y, X)$ and $g \in \operatorname{hom}(X, Z)$, where $Y$ and $Z$ are arbitrary objects in $\mathcal{C}$.[Identity]

Intuitively, a category is what we would expect in a family: a class of objects and operations on these objects, where the operations need to obey the well defined mathematical rules.

Accepting the notion of a part family as a category implies that we can now precisely formulate several important problems in modeling and representing part families. Part families must rely on some topological structure of the part representation, so that we can define some notion of continuity. As illustrated for the two important and well known part families: CSG and b-rep families, the topological structure on a part representation could be either a parametric structure induced by a parametric representation, or the topological structure could be induced by the Euclidean subspace of a part representation. We will 
call any family induced by some parametric topological structure as a parameter controlled family and any family induced by the Euclidean topological structure as a shape controlled family. Note that either concept of family must rely on a part representation and hence will be known collectively as representation induced families. It should be clear that a family can be defined only if we have atleast one object (called the nominal object) from the family, since a topological space is always defined on a given set. This requirement of nominal object is also present in tolerance classes [Requicha, 1977; Stewart, 1992]. A notion of family of solids also exists in the area of geometric tolerancing and robustness [Requicha, 1977; Stewart, 1992]. The definition of a family proposed by [Requicha, 1977] can be thought of as a purely geometric notion since any notion of continuity can be defined only for topological spaces. At the same time, our definitions are consistent and more general than the definition proposed by [Stewart, 1992].

Assuming some topological structure has been assumed on a part representation (unless otherwise stated), we can precisely state the problem of generating objects in a family or testing (classifying) an object with respect to a family. The problem of generating objects in a given part family $\mathcal{F}$ with a nominal part $X$ is equivalent to applying a known morphism $g \in h o m_{\mathcal{F}}$ on $X$. In this paradigm, we know the class (type) of objects $O b_{\mathcal{F}}$ and also the morphism set $\operatorname{hom}_{\mathcal{F}}(X, Y)$ between $X$ and some object $Y$ in the family $\mathcal{F}$. Any family representation defined using this approach can be classified as belonging to the generative paradigm. A key issue in generative paradigm is to develop the precise rules (semantics) for enforcing the morphisms in a given family, so that it always can generate other parts (objects) in the family given one part from it. The alternate problem of testing an arbitrary (untopologized) part $Y$ for membership in a given part family $\mathcal{F}$ with a nominal part $X$, is equivalent to assuming a topology on $Y$ and then constructing an appropriate morphism $g: X \rightarrow Y$. Any family defined using this approach can be classified as belonging to the object membership classification paradigm. The key issue in this paradigm is in assigning a topological structure to $Y$ and then constructing a map $g$ from $X$ to $Y$. The classification paradigm appears harder than generation, due to the fact that the map $g$ between the two parts are not known a priori, till we assign a topological structure on $Y$.

Generating objects in a CSG family with a parametric topological structure is fairly easy, since every set of parameter values (a point in the parametric space) can be assigned a trivial topology and thus making every parametric change as continuous. On the other hand, b-reps usually carry some type of topology as cell complexes in Euclidean spaces and can be assigned different classes of continuous maps (cell maps, orientation preserving cell maps,etc). Due to the separation of embedding (underlying set) from the representation CSG families have a nice parametric control. On the other hand the embedding 
and the representation for b-reps is closely knit, making it difficult to define parameter controlled b-rep families. These complementary properties usually lead to composite families defined on representations that contain the two type of topological structures.

\section{Multiple part families and functors}

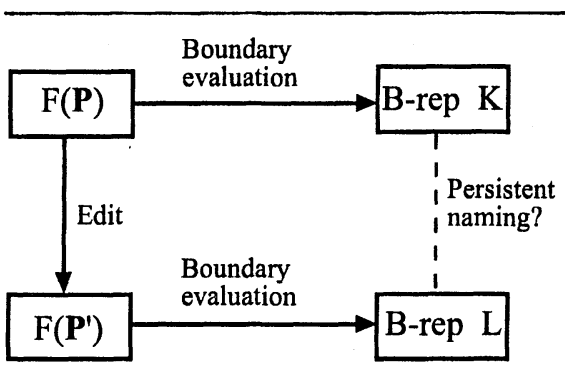

(a) Parametric update through boundary evaluation

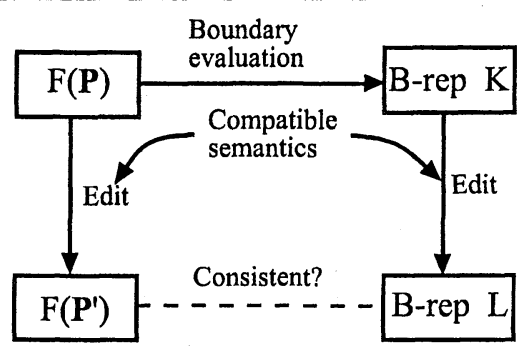

(b) Simultaneous dual representation update

Figure 3. Two approaches for updating objects in composite families

In composite families, there are two approaches of updating the representations for generating other members of the family as illustrated in figure 3 . In the first approach shown in figure 3(a), for every parametric generation $f$ a new b-rep $L$ is computed from the updated parametric representation $F\left(\mathbf{P}^{\prime}\right)$. The problem now is to classify $L$ with respect the family defined on b-rep $K$, i.e, to construct a map $g: K \rightarrow L$, such that it corresponds to the parametric edit $f$. This problem known as the "persistent naming" problem, is currently being solved using heuristic procedures [Kripac, 1997; Capoyleas et al., 1996]. The principle of continuity on b-rep families developed by the authors [S.Raghothama and V.Shapiro, 1998], alleviates this problem to some extent, but does not solve it.

Practical difficulties of "persistent naming" and efficiency reasons, prompted the authors to pursue an alternate approach to modeling composite families and is shown in figure 3(b). Instead of evaluating the b-rep $L$ from $F\left(\mathbf{P}^{\prime}\right)$ again, we simultaneously update both $F(\mathbf{P})$ and $K$ by assuming a compatible semantics (i.e, we assume $f$ is induced by $g$ ). Assuming that the update resulted in a valid b-rep $L$ and that it obeys the two continuity conditions developed by the authors [S.Raghothama and V.Shapiro, 1998], we face a different kind of problem now: we need to verify if the updated parametric definition $F\left(\mathbf{P}^{\prime}\right)$ and the b-rep $L$ are consistent (correspond) with each other or not. For instance, consider the 
b-rep $K$ shown in figure 2(a), that was derived from the CSG representation $F(\mathbf{P})$ shown in figure 1(a). The compatible semantics in this case is the following: changing the parameter values of the position vector $P_{1}$ (i.e, moving the primitive $A$ ), is equivalent to (or induces) a transformation of the face $f_{1}$ by the same translation vector $\left(P_{1}^{\prime}-P_{1}\right)$. However this induced transformation may not result in consistency between $F\left(\mathbf{P}^{\prime}\right)$ and $L$, as illustrated by the update of the b-rep in figure 2(b): the b-rep $L$ is not consistent with $F\left(\mathbf{P}^{\prime}\right)$ due to the fact that both represent different pointsets (figure 1(e) shows the set represented by $F\left(\mathbf{P}^{\prime}\right)$ ). The problem of consistency verification was studied by [S.Raghothama and V.Shapiro, 1999], who also developed algorithms for the consistency verification of CSG and b-rep under simultaneous dual updates. Either way, the main difficulty with multiple families is the incompatibilities arising among them and the lack of precise relationship between any two such families.

The formal relationship between any two categories is captured by the notion of functors. Intuitively, a functor serves as a function between two families by establishing a correspondence between their objects and morphisms, such that the rules on the morphisms are also preserved. The following definition of a functor is adapted from [Munkres, 1984] and [Jänich, 1983].

Definition 2 (Functor) A functor $\varphi$ from a category $\mathcal{C}$ to a category $\mathcal{D}$ is a function assigning to each object $X$ of $\mathcal{C}$, an object $\varphi(X)$ of $\mathcal{D}$, and to each morphism $f: X \rightarrow Y$ of $\mathcal{C}$, a morphism $\varphi(f): \varphi(X) \rightarrow \varphi(Y)$ of $\mathcal{D}$ such that the following conditions are satisfied:

$$
\begin{aligned}
& 1 \varphi\left(1_{X}\right)=1_{\varphi(X)}, \text { for all } X . \\
& 2 \varphi(g \circ f)=\varphi(g) \circ \varphi(f) .
\end{aligned}
$$

Assuming that a composite family is defined as the corresponding pairs of objects and morphisms, let us see how we can formalize the problems in the two architectures for composite families shown in figure 3 using functors. In the first architecture of composite families shown in figure 4(a), we need to deal with two problems:

1 to assign a topology on $L$ and construct a persistent naming map $g: K \rightarrow$ $L ;$ and

2 to verify that $\varphi: f \rightarrow g$.

This implies that the pairs $(F(\mathbf{P}), K),\left(F\left(\mathbf{P}^{\prime}\right), L\right)$ and $(f, g)$ belong to the composite family as given by the definition of a functor. While the correspondence $\varphi$ between objects $F\left(\mathbf{P}^{\prime}\right)$ and $L$ of the two families is established by the ability to convert a parametric representation to a b-rep, the correspondence $\varphi$ between the morphisms $f$ and $g$ cannot be verified unless we assign some topology on $L$ 


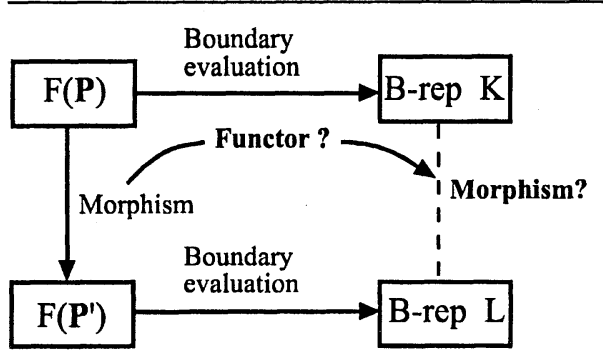

(a)

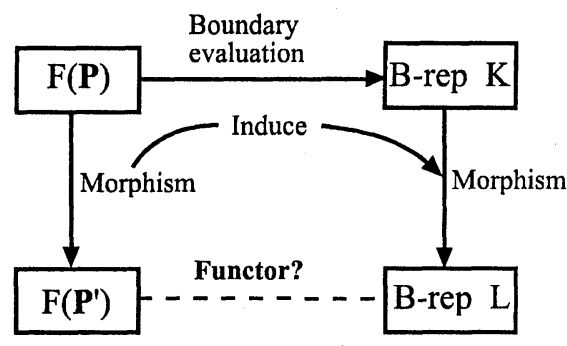

(b)

Figure 4. Defining a composite parametric-brep family (a) Single representation updates requires us to solve two problems: the construction of a morphism $g: K \rightarrow L$ and if the assumed correspondence $\varphi$ between the objects also induces a correspondence $\varphi: f \rightarrow g$ such that $\varphi$ is a functor between the families (b) Dual representation updates requires us to solve one problem: if the assumed correspondence $\varphi: f \rightarrow g$ also induces a correspondence between $L$ and $\left(F\left(\mathbf{P}^{\prime}\right)\right)$ such that $\varphi$ is a functor between the families

and construct a map $g$. It is easy to see that the family $\mathcal{F}(F(\mathbf{P}))$ is based on the generative paradigm and the family $\mathcal{V}(K)$ is based on the object membership classification paradigm, as we always generate objects in $\mathcal{F}(F(\mathbf{P}))$ and compute a new b-rep $L$ (through representation conversion on $F\left(\mathbf{P}^{\prime}\right)$ ) and verify if $L \in \mathcal{V}(K)$.

In the case of second architecture of a composite family shown in figure 4(b), we generate the members in both families by their respective morphisms $f$ and $g$, by assuming that $\varphi: f \rightarrow g$ (i.e, $g$ is induced by $f$ ). We then check if this assumed correspondence $\varphi$ between the morphisms also induces a correspondence $\varphi$ between the resulting objects $F\left(\mathbf{P}^{\prime}\right) \in \mathcal{F}(F(\mathbf{P}))$ and $L \in \mathcal{V}(K)$ for their membership in the composite family, i.e, if $\varphi$ is a functor or not. This correspondence task in turn reduces to verifying consistency between $F\left(\mathbf{P}^{\prime}\right)$ and $L$, as we are looking for a particular type of functor known as the identity functor. It should be easy to infer the complexity of the first approach (shown in figure 4(a)) over the second approach (shown in figure 4(b)): the first approach is harder than the second one since we have to deal with two pairs of topological spaces and maps between in this case, while we just have to deal with two part representations (untopologized instances) of two families in the second approach.

\section{Conclusions}

We identified categories and functors as the proper mathematical models for defining part families and their relationship. These mathematical models al- 
lows us to formulate the various classes of problems in part families: such as generating objects, testing an object against a family, persistent naming and consistency of representations. We also identified two different types of families: parameter controlled and shape controlled families, and argued that both are representation induced families. Parameter controlled families rely on a parametric topological structure induced by a given (nominal) part representation as the objects and continuous maps in the parametric space as the morphisms. At the same time, shape controlled families rely on the Euclidean topological structure induced by the nominal part representation as the objects and the continuous maps in the corresponding topology as the morphisms. We also argued about the advantages of the generative paradigm over the classification paradigm of defining part families.

\section{Acknowledgments}

This research is supported in part by the National Science Foundation CAREER award DMI-9502728, grant DMI-9522806, DARPA contract N0001496-C-0168 and Unigraphics Solutions Corporation.

\section{References}

Borisovich, Y., Bliznyakov, N., Izrailevich, Y., and Fomenko, T. (1985). Introduction to Topology. Mir Publishers, Moscow.

Capoyleas, V., Chen, X., and Hoffmann, C. M. (1996). Generic naming in generative, constraintbased design. Computer-Aided Design, 28(1):17-26.

Jänich, K. (1983). Topology. Springer-Verlag, New York.

Kripac, J. (1997). A mechanism for persistently naming topological entities in history-based parametric solid models. Computer-Aided Design, 29(2):113-122.

Maunder, C. R. F. (1996). Algebraic Topology. Dover Publications, New York.

Munkres, J. R. (1984). Elements of Algebraic Topology. Addison-Wesley, Reading, Massachusetts.

Requicha, A. A. G. (1977). Part and assembly description languages: I - dimensioning and tolerancing. Technical report, TM-19, PAP, University of Rochester, Rochester, NY.

S.Raghothama and V.Shapiro (1998). Boundary representation deformation in parametric solid modeling. ACM Transactions on Graphics, 17(4):259-286.

S.Raghothama and V.Shapiro (1999). Consistent updates in dual representation systems. In $A c$ cepted for publication in 5th ACM Symposium on Solid Modelling and Applications, Ann Arbor, Michigan, June.

Stewart, N. F. (1992). Sufficient condition for correct topological form in tolerance specification. Computer-Aided Design, 12:39-48. 